\title{
Las empresas hospitalarias de Andalucía, Cataluña y la Comunidad de Madrid: Análisis financiero y de sostenibilidad
}

\author{
JUDIT CREIXANS-TENAS ${ }^{a}$, NÚRIA ARIMANY-SERRAT ${ }^{\text {a }}$ \\ a Universidad de Vic-Ucc, Facultad de Empresa y Comunicación, c/ Sagrada Familia, 7, 08500 Vic \\ (Barcelona),España.E-mail: judit.creixans@uvic.cat, nuria.arimany@uvic.cat
}

\begin{abstract}
RESUMEN
El sector sanitario privado representa un importante agente en la sanidad de España, por ser un aliado estratégico del sistema público de salud. En este contexto, Andalucía, Cataluña y la Comunidad de Madrid se posicionan como las tres comunidades autónomas más privatizadas de España. El estudio hace un análisis de los indicadores financieros principales de los centros hospitalarios en el periodo 2008-2015 de estas comunidades autónomas y estudia la relación que tienen los indicadores no financieros relacionados directamente con la Responsabilidad Social Corporativa (RSC) con los resultados económicos y financieros obtenidos. En términos generales, los indicadores financieros de las empresas hospitalarias de Andalucía, Cataluña y la Comunidad de Madrid muestran que a lo largo del período analizado mejoran sus resultados financieros. Además, la rentabilidad financiera de las tres comunidades autónomas se explica por la presencia de indicadores de RSC.
\end{abstract}

Palabras clave: hospitales, sector privado, crisis económica, análisis financiero, RSC.

\section{Hospital Companies of Andalucía, Catalonia and the Community of Madrid: Financial and Sustainability Analysis}

\begin{abstract}
The private health sector represents an important agent in the health of Spain, for being a strategic ally of the public health system. In this context, Andalusia, Catalonia and the Community of Madrid are positioned as the three most privatized autonomous communities in Spain. The study makes an analysis of the main financial indicators of the hospitals in the period 2008-2015 of these autonomous communities and studies the relationship of non-financial indicators directly related to Corporate Social Responsibility (CSR) with economic and financial results obtained. In general terms, the financial indicators of the hospital companies of Andalusia, Catalonia and the Community of Madrid show that their financial results improve during the analysed period. In addition, the financial profitability of the three autonomous communities is explained by the presence of CSR indicators.
\end{abstract}

Keywords: Hospitals, Private Sector, Economic Crisis, Financial Analysis, CSR.

Clasificación JEL: I18, M21

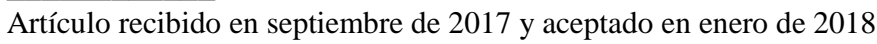

Artículo disponible en versión electrónica en la página www.revista-eea.net, ref. ə-36202 


\section{INTRODUCCIÓN}

El análisis económico y financiero de los centros hospitalarios en el periodo 2008-2015, caracterizado por la recesión económica, interesa especialmente ya que contempla la salud de los servicios sanitarios con un importante papel social.

Dichos servicios sanitarios, en España, dependen de las Comunidades Autónomas, y el presupuesto sanitario lo fijan cada año los parlamentos autonómicos, descontándose éste de la partida general recibida del gobierno central.

De hecho, una de las transformaciones más relevantes que ha realizado a lo largo de los años el gobierno, en términos de sanidad, se ha producido con la transferencia de la gestión de la asistencia sanitaria a las comunidades autónomas (Meneu y Ortún, 2011).

De acuerdo con el informe del año 2017, elaborado por el Instituto para el Desarrollo e Integración de la Sanidad (2017), en algunas comunidades autónomas se intenta facilitar la intervención de aseguradoras privadas para proveer de más servicios sanitarios complementarios al sistema público de salud.

Así pues, el sector sanitario privado representa un importante agente en la sanidad de España, por ser un aliado estratégico del sistema público de salud y por disponer de un 57\% de los todos los centros hospitalarios españoles.

En este contexto, cabe destacar que Andalucía, Cataluña y la Comunidad de Madrid se posicionan como las tres comunidades autónomas más privatizadas de España ya que concentran el 57\% de los hospitales privados y el $62 \%$ de las camas hospitalarias privadas.

Así, el estudio pretende posicionar y comparar los centros analizados de estas comunidades autónomas en el sistema sanitario español y presentar los principales indicadores para diagnosticar la salud económica y financiera en este periodo mediante un análisis a corto y largo plazo, un análisis de resultados y un análisis de los cambios patrimoniales y de los flujos de efectivo.

Además, se analizan indicadores no financieros relacionados directamente con la Responsabilidad Social Corporativa, por la importancia de que estas entidades hospitalarias dispongan de acreditaciones y memorias de sostenibilidad para el correcto desarrollo de sus actividades. Es por eso que se pretende determinar si existe una relación significativa entre estos indicadores y los resultados económicos y financieros obtenidos.

\section{MARCO TEÓRICO}

La situación económica financiera de la sanidad española ha cambiado mucho a lo largo de las últimas décadas debido al contexto de crisis sistémica 
española generalizada. Además, dicha situación preocupa a los principales responsables del ámbito público y privado.

En concreto, aunque todavía no se puede evaluar en profundidad el impacto de la crisis, hay evidencias que demuestran que impacta en la sanidad pública y en los servicios sanitarios privados (Cortés-Franch y López-Valcárcel, 2014).

En la actualidad los desafíos de la gestión hospitalaria pública y privada deben contemplar especialmente el análisis de los estados financieros en los nuevos modelos de gestión hospitalaria (Calvo, 2007) y hay estudios que detallan la necesidad de reportar indicadores financieros en los hospitales (Pink y otros, 2007).

Además, se debe tener presente que la sanidad privada tiene que estar en sintonía con un sistema público de salud (López, 2009) y que la transparencia y el buen gobierno en la sanidad ayudaran a salir de la crisis (Meneu y Ortún, 2011).

\subsection{Indicadores financieros}

El análisis de los estados financieros se configura como un conjunto de técnicas útiles para que toda empresa pueda tomar las decisiones adecuadas a su situación y perspectiva económica y financiera (Amat, 2000).

Son muy pocos los estudios que analizan los estados financieros de las empresas hospitalarias españolas (Creixans-Tenas y Arimany-Serrat, 2016; Giner y otros, 2005). No obstante, en otros países es común la utilización de indicadores financieros en forma de ratios para establecer la situación económica y financiera de las empresas hospitalarias (Lee y Choi, 2011).

Pink y otros (2007) definieron en su estudio los mejores indicadores financieros para determinar la posición financiera y económica de las empresas que forman parte del sector hospitalario. Particularmente, establecieron como variables principales a analizar: la rentabilidad financiera, la rentabilidad económica, la solvencia a corto plazo, el fondo de maniobra, la capitalización y el endeudamiento.

Según Cortés y otros (2011), en los últimos años, debido a la crisis económica, se ha hecho más relevante analizar la rentabilidad financiera, junto con variables que la definen mejor, para poder disponer de herramientas que permitan obtener una mejor planificación financiera a largo plazo.

\subsection{Indicadores no financieros}

La Responsabilidad Social Corporativa (RSC) se configura como un elemento estratégico clave en la gestión de cualquiera empresa (Jiménez y otros, 2014) y se define como aquel proceso en el cual las empresas se implican para conseguir una mejor sociedad y un medio ambiente más eficiente (Valenzuela y otros, 2015) 
En este ámbito, la RSC engloba cinco dimensiones diferenciadas: económica, legal, social, ética y medioambiental y cada año aumentan las organizaciones que informan sobre cuestiones relativas a estas dimensiones (Frías-Aceituno y otros, 2013; Marín-Calahorro, 2008; Prado-Lorenzo y García-Sánchez, 2010).

En los últimos años, la población ha mostrado un mayor interés por la transparencia de las entidades y su sostenibilidad (Nevado-Gil y GallardoVázquez, 2016) y hay más centros hospitalarios que adoptan buenas prácticas en materia de RSC (Rodríguez-Cala y otros, 2015).

Los indicadores de RSC se reflejan mediante las acreditaciones medioambientales o de calidad y las memorias de sostenibilidad (Satorras, 2002) y la página web corporativa de los hospitales es un medio útil para mostrar más transparencia y destacar la sostenibilidad y posicionarse mejor respecto la competencia (Rodríguez-Cala y otros, 2015). De hecho, Camacho y otros (2013) estructuraron las herramientas que han sido desarrolladas en materia de RSC, clasificándolas en principios, instrumentos e indicadores.

\subsection{Relación entre rentabilidad e indicadores no financieros}

Son muchos los estudios científicos que se configuran alrededor de la relación existente entre RSC y rentabilidad financiera (Miras y otros, 2011; Navarro y González, 2006). Lo más habitual en los estudios precedentes es que se considere la RSC como variable independiente (Alonso-Almeida y otros, 2012; Charlo y Moya, 2010).

Ya en los años 90, Cleverley (1990) manifestó que la información no financiera combinada con las ratios financieras oportunos y, particularmente con la rentabilidad financiera, aportan un mayor valor añadido para poder diagnosticar la salud de las empresas hospitalarias.

En el trabajo de Gómez (2008) se analizan las posibles relaciones existentes entre ambas variables y se establece que el signo de dicha relación puede ser positiva, neutral o negativa.

No obstante, la relación existente entre RSC y la rentabilidad financiera está determinada por el sector de actividad que se analiza (Jiménez y otros, 2014).

Concretamente para el caso de las entidades hospitalarias, hay autores que manifiestan que existe una relación positiva entre ambas variables (Antunes y Gallardo, 2016) y otros que exponen que, aunque con acreditaciones y memorias de sostenibilidad mejoran los indicadores financieros, no existe un criterio unificado para asegurar este dato (Setó y Angla, 2011).

\section{OBJETIVOS E HIPÓTESIS}

De acuerdo con la literatura previa analizada, el objetivo principal de esta investigación es posicionar, comparar y diagnosticar la salud económica y 
financiera de las empresas hospitalarias de las comunidades autónomas más privatizadas - Andalucía, Cataluña y la Comunidad de Madrid (mediante un análisis convencional a corto y largo plazo, un análisis de resultados y un análisis de los cambios patrimoniales).

Además, se analizan indicadores no financieros relacionados directamente con la Responsabilidad Social Corporativa para determinar si existe una relación significativa entre las rentabilidades financieras de cada comunidad autónoma y los indicadores no financieros obtenidos.

Concretamente, se plantean las hipótesis siguientes:

H1: Existe una relación significativa entre los indicadores de RSC y la rentabilidad financiera de las empresas hospitalarias de Andalucía.

H2: Existe una relación significativa entre los indicadores de RSC y la rentabilidad financiera de las empresas hospitalarias de Cataluña.

H3: Existe una relación significativa entre los indicadores de RSC y la rentabilidad financiera de las empresas hospitalarias de la Comunidad de Madrid.

\section{METODOLOGÍA}

Para la realización de esta investigación se ha utilizado la información contable y financiera presentada en el Registro Mercantil por las empresas y recogida en la base de datos SABI.

Concretamente, la metodología ha consistido en realizar el análisis económico y financiero de tres muestras de empresas hospitalarias españolas en el período 2008-2015, atendiendo a los siguientes criterios de búsqueda:

- Empresas mercantiles.

- Empresas activas.

- Período de tiempo: 2008-2015.

- Sector de actividad: actividades hospitalarias (CNAE 2009: 8610).

- Empresas grandes y medianas: atendiendo a la Directiva Europea 2013/34/UE se identifican como grandes y medianas empresas aquellas que el importe neto de la cifra de negocios y el activo total superan los 8 y 4 millones de euros, respectivamente, y que disponen de más de 50 trabajadores.

La muestra obtenida está formada por 44 empresas hospitalarias, cuya facturación supone un $62 \%$ del total de las empresas hospitalarias españolas. De estas empresas, tal y como se observa en la primera Figura, 11 pertenecen a la comunidad autónoma de Andalucía, 15 a Cataluña y las 18 restantes a la Comunidad de Madrid. 
Figura 1

Distribución geográfica de las empresas hospitalarias

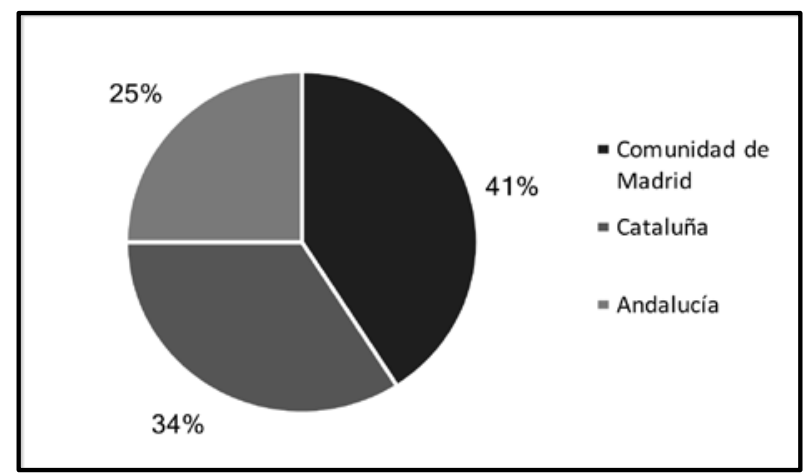

Fuente: Elaboración propia.

Una vez establecidas las muestras, se han calculado los indicadores financieros correspondientes y se ha realizado el análisis económico y financiero convencional a corto y a largo plazo.

Concretamente, para realizar este análisis convencional, los indicadores utilizados han sido: fondo de maniobra, solvencia a corto plazo, endeudamiento, calidad de la deuda, rentabilidades y apalancamiento financiero.

A continuación, de acuerdo con la literatura previa analizada y las hipótesis planteadas, para el último ejercicio económico se ha relacionado la rentabilidad financiera de cada una de las comunidades autónomas con la variable explicativa correspondiente a los indicadores de RSC.

Para relacionar dichas variables se han utilizado contrastes de hipótesis mediante estadística inferencial y utilizando el programa estadístico IBM SPSS Statistics en su versión 22. Concretamente, se han realizado las pruebas de normalidad y de homogeneidad y para terminar la realización de la prueba $T$ de Student para comparar las medias y así dar respuesta a las hipótesis planteadas (Nevado-Gil y Gallardo-Vázquez, 2016).

De este modo, se ha establecido como variable dependiente la rentabilidad financiera (ROE), es decir, la relación entre el beneficio económico y los recursos necesarios para obtener dicho beneficio. En definitiva, son los beneficios obtenidos por invertir dinero en recursos financieros. Dicha rentabilidad se ha calculado relacionando el resultado del ejercicio con los fondos propios de cada una de las empresas hospitalarias que forman las tres muestras.

Como variable explicativa se ha tomado la presencia de indicadores de RSC en cada una de los centros hospitalarios. Esta variable se ha definido como categórica de la siguiente forma: 
- Si la empresa hospitalaria dispone de memorias de sostenibilidad siguiendo los criterios del Global Reporting Initiative (GRI), acreditaciones ISO 9.001, 14.001, 26.000 o 50.001, o acreditaciones de calidad EFQM (European Foundation for Quality Management) o JCI (Joint Commission International) en sus páginas web (Satorras, 2002): la variable toma el valor 1.

- En caso que la empresa hospitalaria no disponga de ninguno de estos indicadores: la variable toma el valor 0 .

Se debe tener en cuenta que para buscar dichos indicadores se han consultado, a lo largo del mes de enero de 2016, los sitios web de cada uno de los centros hospitalarios que definen las tres muestras.

\section{CARACTERIZACIÓN}

Para definir y posicionar correctamente a las empresas hospitalarias analizadas de las tres Comunidades Autónomas más privatizadas, se ha realizado una caracterización mediante los datos que hacen referencia a la forma jurídica, la fecha de constitución y la clasificación entre grande o mediana empresa.

\subsection{Forma jurídica}

Tal y como se observa en las Figuras 2, 3 y 4, en el caso de Andalucía y la Comunidad de Madrid, el número de sociedades anónimas es superior al de sociedades limitadas; en cambio, en Cataluña la proporción de ambas sociedades es prácticamente la misma.

Figura 2

Forma jurídica de las empresas hospitalarias de Andalucía

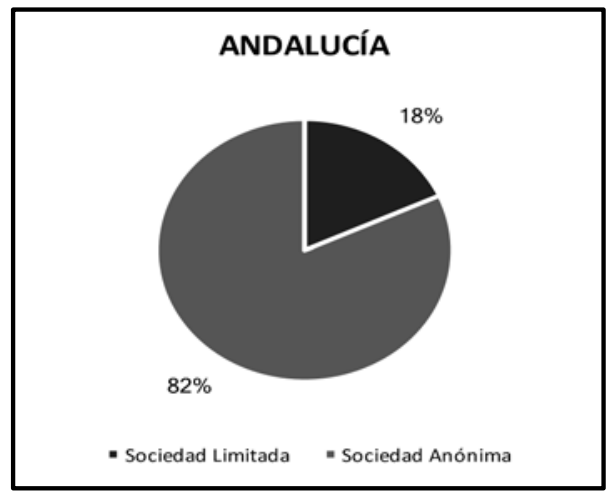

Fuente: Elaboración propia. 
Figura 3

Forma jurídica de las empresas hospitalarias de Cataluña

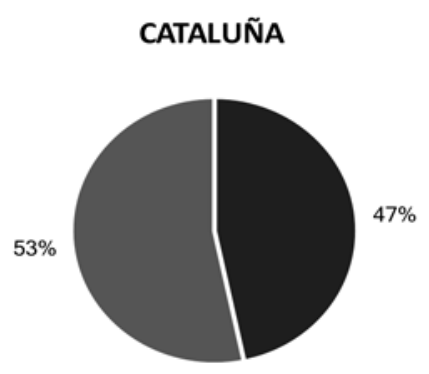

- Sociedad Limitada = Sociedad Anónima

Fuente: Elaboración propia.

Figura 4

Forma jurídica de las empresas hospitalarias de la Comunidad de Madrid

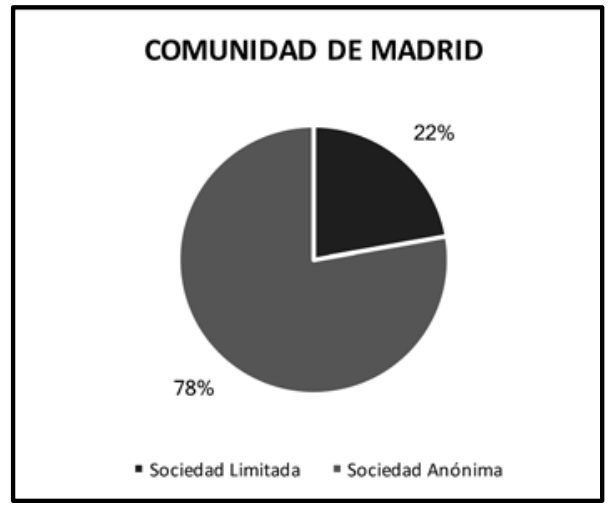

Fuente: Elaboración propia.

\subsection{Fecha de constitución}

En el caso de Andalucía, un 82\% de las empresas se constituyeron hace más de 20 años y sólo una de ellas se ha creado a lo largo del período analizado.

En Cataluña, también sólo una empresa se ha creado a lo largo del período 2008-2015 y un 60\% de las empresas se han constituido hace más de 20 años.

Por último, en la Comunidad de Madrid son más las empresas que se han constituido a lo largo del periodo analizado, concretamente 4. Además, en este caso tan sólo un 32\% fueron constituidas hace más de 20 años. 


\subsection{Clasificación por tamaño}

Si se observan las Figuras 5, 6 y 7, en las tres comunidades autónomas la presencia de empresas medianas es superior, siguiendo la Directiva Europea 2013/34/UE. No obstante, cabe destacar que, en la comunidad de Madrid, el porcentaje de empresas grandes es mayor al de las otras dos comunidades.

Figura 5

Clasificación por tamaño de las empresas hospitalarias de Andalucía

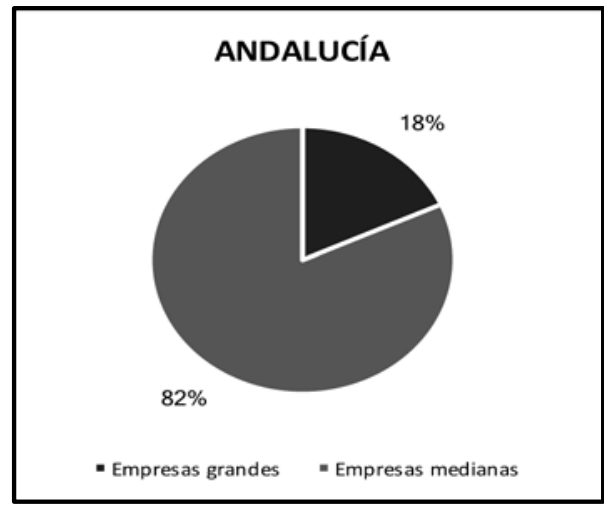

Fuente: Elaboración propia.

Figura 6

Clasificación por tamaño de las empresas hospitalarias de Cataluña

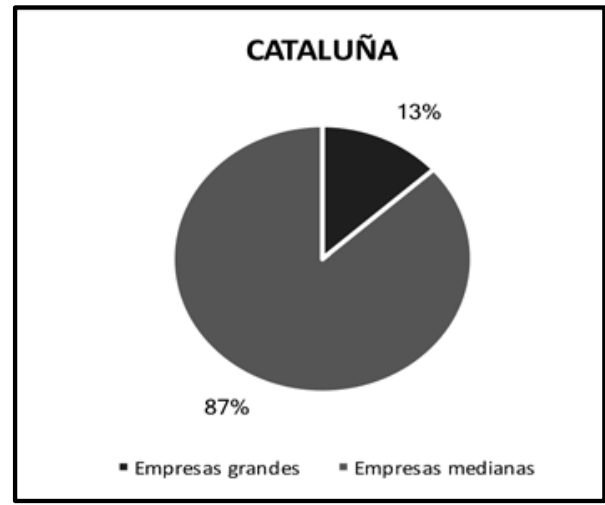

Fuente: Elaboración propia. 
Figura 7

Clasificación por tamaño de las empresas hospitalarias de la Comunidad de Madrid

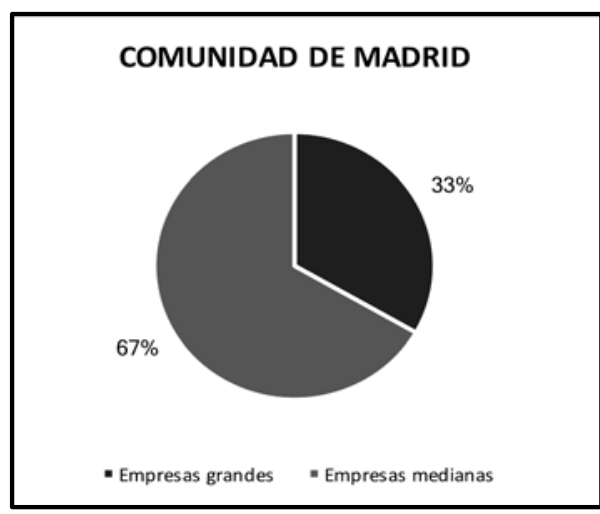

Fuente: Elaboración propia.

\section{RESULTADOS}

\subsection{Análisis de la situación económica y financiera}

El análisis de la situación económica y financiera en el período 2008-2015 de las empresas hospitalarias de las comunidades autónomas de Andalucía, Cataluña y Madrid, comprende el estudio de los principales indicadores financieros.

Respecto al análisis de la situación financiera a corto plazo, en la Tabla 1 se observa que el fondo de maniobra es positivo en todos los períodos, exceptuando el 2008 de la muestra de la Comunidad de Madrid, además aumenta a lo largo del período analizado, aunque sus valores sean inferiores a las otras dos comunidades.

\section{Tabla 1}

Fondo de maniobra

(miles de euros)

\begin{tabular}{|c|c|c|c|c|c|c|c|c|}
\hline & $\mathbf{2 0 0 8}$ & $\mathbf{2 0 0 9}$ & $\mathbf{2 0 1 0}$ & $\mathbf{2 0 1 1}$ & $\mathbf{2 0 1 2}$ & $\mathbf{2 0 1 3}$ & $\mathbf{2 0 1 4}$ & $\mathbf{2 0 1 5}$ \\
\hline ANDALUCÍA & 8.890 & 11.287 & 9.882 & 13.024 & 12.342 & 14.805 & 17.098 & 18.825 \\
\hline CATALUÑA & 3.645 & 10.271 & 12.350 & 11.929 & 16.387 & 10.497 & 10.249 & 10.485 \\
\hline MADRID & -3.594 & 1.977 & 1.544 & 199 & 2.779 & 2.840 & 1.821 & 3.060 \\
\hline
\end{tabular}

Fuente: Elaboración propia.

Se debe tener en cuenta que la solvencia media a corto plazo de las empresas de Andalucía y Cataluña es elevada, con tendencia a aumentar a lo largo del periodo analizado, hecho que puede inducir a que las empresas tengan activos circulantes ociosos. Por su parte, la Comunidad de Madrid obtiene una solvencia a corto plazo moderada, por debajo del resultado de referencia (entre 1,5 y 2) y es 
preciso concretar que, en el año 2008, coincidiendo con el fondo de maniobra negativo, era relativamente baja (Tabla 2).

Tabla 2

Solvencia a corto plazo

\begin{tabular}{|c|c|c|c|c|c|c|c|c|}
\hline & $\mathbf{2 0 0 8}$ & $\mathbf{2 0 0 9}$ & $\mathbf{2 0 1 0}$ & $\mathbf{2 0 1 1}$ & $\mathbf{2 0 1 2}$ & $\mathbf{2 0 1 3}$ & $\mathbf{2 0 1 4}$ & $\mathbf{2 0 1 5}$ \\
\hline ANDALUCíA & 2,69 & 2,84 & 2,70 & 3,09 & 2,64 & 3,06 & 3,30 & 3,39 \\
\hline CATALUÑA & 1,77 & 2,27 & 2,29 & 1,97 & 2,52 & 2,20 & 2,19 & 2,38 \\
\hline MADRID & 0,79 & 1,13 & 1,10 & 1,01 & 1,16 & 1,14 & 1,09 & 1,15 \\
\hline
\end{tabular}

Fuente: Elaboración propia.

Por lo que hace referencia al análisis de la situación financiera a largo plazo, las Tablas 3 y 4 muestran que las empresas hospitalarias que están más endeudadas son las de la Comunidad de Madrid, con un endeudamiento que aumenta a lo largo de los años y supera la franja de endeudamiento normal (50\%60\%). En cambio, los centros hospitalarios de Andalucía tienen un bajo endeudamiento que se mantiene a lo largo del periodo analizado.

No obstante, la calidad de la deuda es mejor en las empresas hospitalarias de la Comunidad de Madrid, aunque cabe destacar la mejora de dicha calidad en los centros de Cataluña al final del periodo analizado. Es importante concretar que en las tres comunidades autónomas las deudas con las entidades de crédito sólo suponen alrededor del 5\% del total del pasivo.

Tabla 3

Endeudamiento

\begin{tabular}{|c|c|c|c|c|c|c|c|c|}
\hline & $\mathbf{2 0 0 8}$ & $\mathbf{2 0 0 9}$ & $\mathbf{2 0 1 0}$ & $\mathbf{2 0 1 1}$ & $\mathbf{2 0 1 2}$ & $\mathbf{2 0 1 3}$ & $\mathbf{2 0 1 4}$ & $\mathbf{2 0 1 5}$ \\
\hline ANDALUCÍA & $24,89 \%$ & $34,65 \%$ & $26,67 \%$ & $25,48 \%$ & $29,57 \%$ & $25,31 \%$ & $23,99 \%$ & $23,24 \%$ \\
\hline CATALUÑA & $44,57 \%$ & $35,37 \%$ & $35,70 \%$ & $45,62 \%$ & $41,63 \%$ & $42,79 \%$ & $42,18 \%$ & $41,12 \%$ \\
\hline MADRID & $66,56 \%$ & $68,77 \%$ & $65,31 \%$ & $69,71 \%$ & $69,00 \%$ & $71,45 \%$ & $74,42 \%$ & $77,31 \%$ \\
\hline
\end{tabular}

Fuente: Elaboración propia.

Tabla 4

Calidad de la deuda

\begin{tabular}{|c|c|c|c|c|c|c|c|c|}
\hline & $\mathbf{2 0 0 8}$ & $\mathbf{2 0 0 9}$ & $\mathbf{2 0 1 0}$ & $\mathbf{2 0 1 1}$ & $\mathbf{2 0 1 2}$ & $\mathbf{2 0 1 3}$ & $\mathbf{2 0 1 4}$ & $\mathbf{2 0 1 5}$ \\
\hline ANDALUCÍA & $79,84 \%$ & $58,71 \%$ & $79,65 \%$ & $73,90 \%$ & $72,24 \%$ & $76,65 \%$ & $77,86 \%$ & $75,99 \%$ \\
\hline CATALUÑA & $79,67 \%$ & $85,51 \%$ & $84,59 \%$ & $70,56 \%$ & $61,34 \%$ & $61,55 \%$ & $61,93 \%$ & $54,48 \%$ \\
\hline MADRID & $52,79 \%$ & $44,55 \%$ & $44,86 \%$ & $42,04 \%$ & $39,09 \%$ & $43,21 \%$ & $40,11 \%$ & $39,74 \%$ \\
\hline
\end{tabular}

Fuente: Elaboración propia.

En el análisis de las rentabilidades se observan comportamientos distintos entre las muestras. No obstante, en todos los casos el período 2010-2011 es el 
más afectado por los recortes y el periodo de austeridad en que se vio inmerso el sector sanitario.

En el caso de Andalucía (Figura 8), se observa que ambas rentabilidades decrecen en el período analizado, aunque a partir del año 2010 hay una tendencia a la estabilidad. En todo el período la rentabilidad financiera (ROE) se sitúa por encima de la rentabilidad económica (ROA), de manera que el endeudamiento no perjudica a los centros hospitalarios analizados. De hecho, el apalancamiento financiero es superior a la unidad en todos los ejercicios.

En Cataluña, se observa la fuerte caída de las rentabilidades experimentada en el periodo 2009-2012. Además, en el ejercicio 2011 la ROA es superior a la ROE, de manera que las empresas se ven perjudicadas por el endeudamiento, hecho que se refleja en el apalancamiento financiero inferior a la unidad en este ejercicio (Figura 9).

Tal y como se muestra en la Figura 10, la Comunidad de Madrid obtiene rentabilidades negativas en los dos primeros ejercicios y a partir del año 2010 se recupera notablemente para después estabilizarse. En estos dos ejercicios es cuando la ROA está muy por encima de la ROE, denotando que el endeudamiento perjudica a las empresas (corroborado por el apalancamiento financiero inferior a la unidad).

Figura 8

Rentabilidad financiera y económica de las empresas hospitalarias de Andalucía

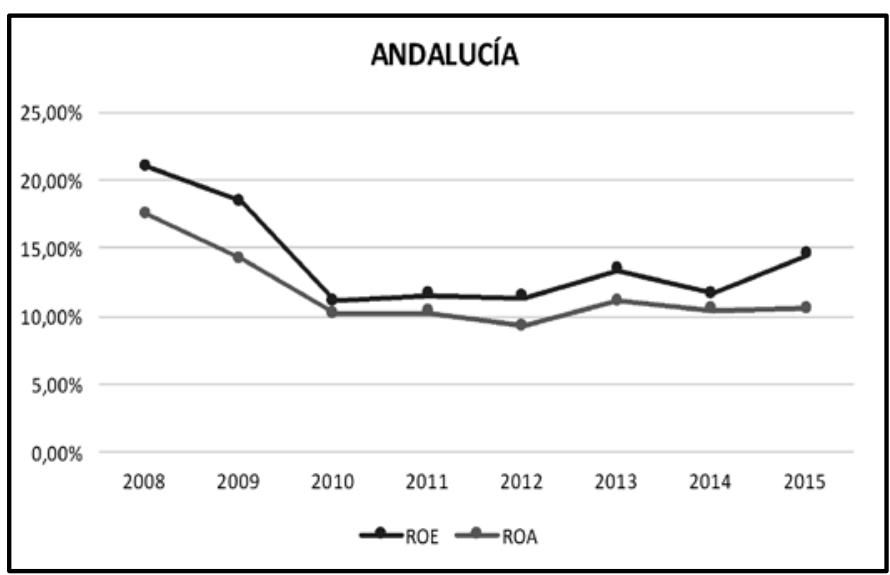

Fuente: Elaboración propia. 
Figura 9

Rentabilidad financiera y económica de las empresas hospitalarias de Cataluña

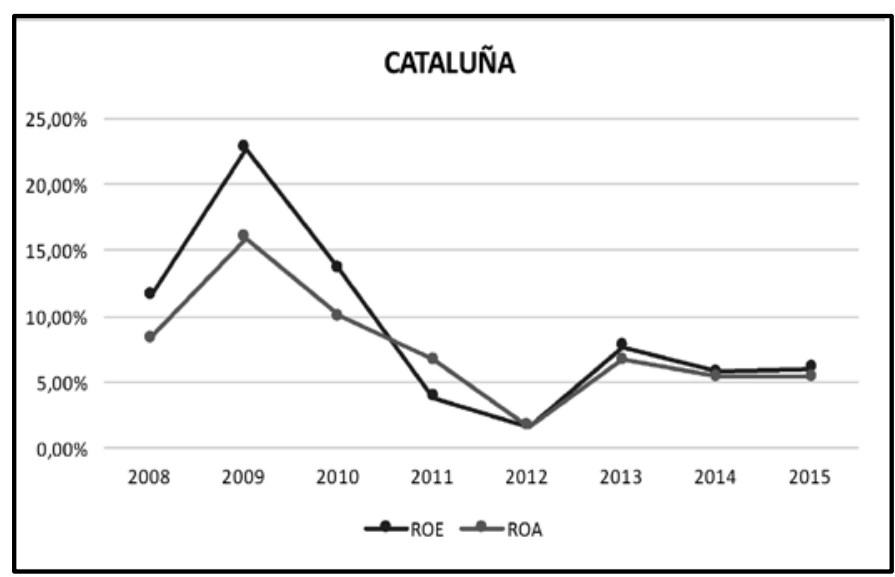

Fuente: Elaboración propia.

Figura 10

Rentabilidad financiera y económica de las empresas hospitalarias de la Comunidad de Madrid

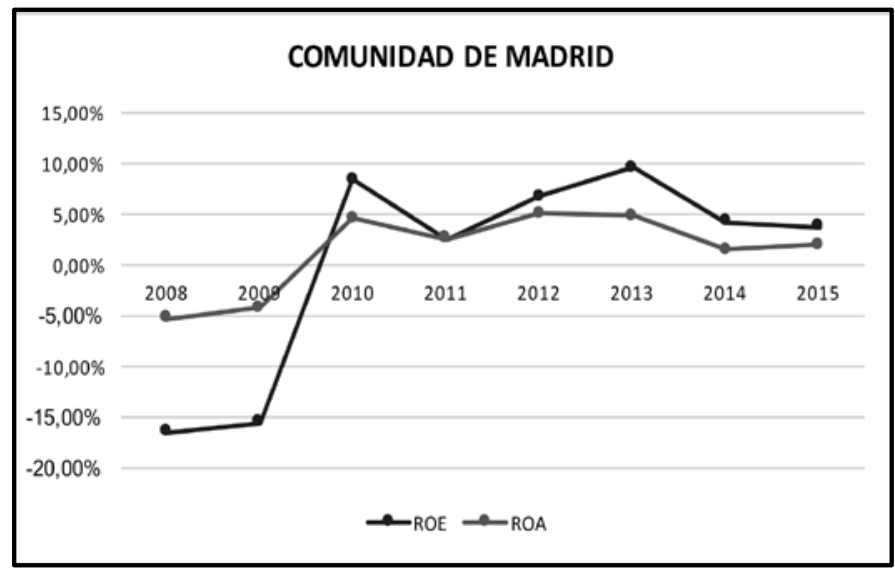

Fuente: Elaboración propia.

Por último, en las tres muestras las empresas hospitalarias están aumentando el patrimonio neto, denotando que dichas empresas se están capitalizando. Concretamente, esta masa patrimonial aumenta un $72 \%$, un $168 \%$ y un 5\%, en Andalucía, Cataluña y la Comunidad de Madrid, respectivamente, debido al aumento del importe del resultado neto. 


\subsection{Relación entre la ROE y los indicadores de RSC}

En este apartado se utilizan contrastes de hipótesis utilizando métodos estadísticos para variables dicotómicas. Concretamente, se pretende contrastar si la presencia de indicadores de RSC en los sitios webs de las empresas hospitalarias de cada una de las tres Comunidades Autónomas influye en el resultado obtenido de la rentabilidad financiera (ROE). Para llevarlo a cabo, se ha realizado un análisis de diferencia de medias de la rentabilidad financiera en función de si dispone o no de indicadores de RSC por cada una de las tres muestras.

Esta prueba estadística requiere que todas las variables cumplan con los supuestos de normalidad y homogeneidad. De este modo, se ha realizado la prueba estadística de Shapiro-Wilk y la prueba de Levene y se llega a la conclusión que todas las variables, tal y como se muestra en la Tabla 5, cumplen los supuestos de normalidad ( $>>0,05$ ), de manera que se realizará el test $T$ Student para muestras independientes.

Tabla 5

Prueba de normalidad de las variables

\begin{tabular}{|c|c|c|c|c|}
\hline \multicolumn{5}{|c|}{ SHAPIRO-WILK } \\
\hline & INDICADORES RSC & Estadístico & gl & $\boldsymbol{p}$ \\
\hline \multirow{2}{*}{$\begin{array}{c}\text { ROE } \\
\text { ANDALUCíA }\end{array}$} & Sí & 0,843 & 6 & 0,139 \\
\cline { 2 - 5 } $\begin{array}{c}\text { ROE } \\
\text { CATALUÑA }\end{array}$ & NO & 0,831 & 5 & 0,142 \\
\cline { 2 - 5 } & Sí & 0,206 & 6 & 0,443 \\
\hline $\begin{array}{c}\text { ROE } \\
\text { MADRID }\end{array}$ & NO & 0,277 & 9 & 0,273 \\
\cline { 2 - 5 } & NO & 0,967 & 11 & 0,602 \\
\hline
\end{tabular}

Fuente: Elaboración propia.

Además, en la Tabla 6 se observa que todas las variables cumplen con el criterio de homogeneidad de varianzas, mediante el uso de la prueba de Levene $(\mathrm{p}>0,05), y$, por lo tanto, para dichas variables se asume que existe igualdad de varianzas para la determinación del estadístico t.

Una vez obtenidos los resultados del contraste de hipótesis mediante la prueba estadística $T$ de Student, se confirma que la presencia de indicadores de RSC en las páginas webs de las empresas hospitalarias de las tres muestras, tiene influencia significativa en las rentabilidades financieras.

Además, los hospitales que disponen de dichos indicadores en sus sitios web presentan un mayor porcentaje de la rentabilidad financiera (Tabla 6). 
Tabla 6

Estadística inferencial

\begin{tabular}{|c|c|c|c|c|c|c|c|}
\hline \multirow{2}{*}{\multicolumn{2}{|c|}{ RENTABILIDAD FINANCIERA }} & \multicolumn{2}{|c|}{ HOMOGENEIDAD } & \multicolumn{2}{|c|}{ ESTADÍSTICOS } & \multicolumn{2}{|c|}{ T-STUDENT } \\
\hline & & Levene & $\mathbf{p}$ & Media & Desviación & $\mathbf{t}$ & $p$ \\
\hline \multirow{2}{*}{$\begin{array}{l}\text { INDICADORES RSC } \\
\text { ANDALUCÍA }\end{array}$} & sí & \multirow{2}{*}{0,478} & \multirow{2}{*}{0,507} & $31,19 \%$ & $24,15 \%$ & \multirow{2}{*}{$-2,289$} & \multirow{2}{*}{0,048} \\
\hline & $\mathrm{NO}$ & & & $1,45 \%$ & $17,52 \%$ & & \\
\hline \multirow{2}{*}{$\begin{array}{c}\text { INDICADORES RSC } \\
\text { CATALUÑA }\end{array}$} & sí & \multirow{2}{*}{0,392} & \multirow{2}{*}{0,542} & $16,03 \%$ & $7,95 \%$ & \multirow{2}{*}{$-4,057$} & \multirow{2}{*}{0,001} \\
\hline & NO & & & $0,34 \%$ & $6,93 \%$ & & \\
\hline \multirow{2}{*}{$\begin{array}{c}\text { INDICADORES RSC } \\
\text { MADRID }\end{array}$} & sí & \multirow{2}{*}{0,123} & \multirow{2}{*}{0,730} & $18,19 \%$ & $26,71 \%$ & \multirow{2}{*}{$-2,848$} & \multirow{2}{*}{0,012} \\
\hline & $\mathrm{NO}$ & & & $2,64 \%$ & $16,27 \%$ & & \\
\hline
\end{tabular}

Fuente: Elaboración propia.

\section{DISCUSIÓN Y CONCLUSIONES}

Después del análisis de los principales indicadores financieros en el período 2008-2015 de las empresas hospitalarias de Andalucía, Cataluña y la Comunidad de Madrid, los resultados evidencian las conclusiones siguientes:

Respecto al análisis a corto plazo, los fondos de maniobra positivos en todas las comunidades autónomas ponen de manifiesto que las empresas pueden hacer frente a sus obligaciones a corto plazo. Se debe tener en cuenta que Andalucía es la Comunidad Autónoma que tiene un fondo de maniobra más elevado y que la Comunidad de Madrid es la que experimenta un comportamiento menos equilibrado del fondo de maniobra, con una caída considerable en el ejercicio 2011. Además, cabe destacar que en Andalucía y Cataluña la solvencia a corto plazo es elevada, con tendencia a aumentar a lo largo del periodo analizado, hecho que puede inducir a que las empresas tengan activos circulantes ociosos. Por su parte, la Comunidad de Madrid obtiene una solvencia a corto plazo moderada, mejorando a lo largo de los ejercicios, hecho que demuestra que la situación a corto plazo ha sido más complicada en esta comunidad autónoma.

En el análisis a largo plazo destaca el bajo endeudamiento de los centros hospitalarios de Andalucía que se sitúa entre el 20\% y el 30\% a lo largo del período analizado, el moderado endeudamiento de los hospitales catalanes, que no alcanza el 50\%, y el elevado endeudamiento de los centros de la Comunidad de Madrid, que alcanza el 77\% en 2015. No obstante, en esta última comunidad la calidad de la deuda es mejor, puesto que no supera el $40 \%$ en el último ejercicio. En Andalucía, dicha calidad de la deuda se mantiene estable en valores que oscilan alrededor del $75 \%$. Mientras que en Cataluña, las deudas a corto plazo significan el 55\% del total de las deudas después de experimentar una considerable mejoría a lo largo de los años.

En el análisis de resultados, la comunidad autónoma que presenta mejores rentabilidades a lo largo del período analizado es Andalucía, ya que se mantiene estable a lo largo del período y en todos los años la ROE supera a la ROA, hecho 
que corrobora que el endeudamiento no perjudica a estas empresas. Por otra parte, en Cataluña, se observa la fuerte caída de las rentabilidades y en el ejercicio 2011 la ROA es superior a la ROE, identificando problemas de endeudamiento en este difícil periodo que es el más afectado por los recortes y la austeridad en que se vio inmerso el sector sanitario Las empresas hospitalarias de la Comunidad de Madrid obtienen rentabilidades negativas en los dos primeros ejercicios y en esos ejercicios la ROA también supera a la ROE. Además, en esta última comunidad autónoma, las rentabilidades tienen un comportamiento menos estable.

En el análisis patrimonial, en las tres muestras las empresas hospitalarias se capitalizan, siendo las de la comunidad autónoma de Cataluña las que muestran un mayor crecimiento de su patrimonio neto, debido al aumento del resultado neto y al aumento del capital para poder realizar las oportunas innovaciones tecnológicas.

En términos generales, los indicadores financieros de las empresas hospitalarias de Andalucía, Cataluña y la Comunidad de Madrid muestran que a lo largo del tiempo mejoran sus resultados, después de la difícil situación de recesión y austeridad provocada por la crisis económica. No obstante, la comunidad autónoma que ha tenido más dificultades para hacer frente a la situación económica soportada en los años 2008-15 ha sido la Comunidad de Madrid. En cambio, Andalucía ha sido la comunidad que ha tenido un comportamiento más estable a lo largo de los años de crisis económica y financiera.

Por último, la rentabilidad financiera de las tres comunidades autónomas se explica por la presencia de indicadores de RSC, en sintonía con la revisión de la literatura (Bem y otros, 2014, 2015; Benton, 2013; Luna y otros, 2009). Concretamente, existe una influencia positiva de los indicadores de RSC (Antunes y Gallardo, 2016). Tal y como se observa, mediante la aplicación de la estadística inferencial, en las tres comunidades autónomas, las empresas hospitalarias que tienen indicadores de RSC en sus páginas web tienen una rentabilidad financiera media muy superior a las empresas hospitalarias que no disponen de dichos indicadores.

Finalmente, de acuerdo con Amat (2000), como limitación de la investigación hay que tener presente que el análisis económico y financiero se basa en datos históricos y puede faltar perspectiva para saber hacia dónde se dirigen las empresas hospitalarias. Además, de acuerdo con Jiménez y otros (2009), los resultados de este estudio que hacen referencia a los indicadores de RSC disponen de una validez temporal, puesto que los sitios webs pueden modificarse en cualquier momento. 


\section{REFERENCIAS BIBLIOGRÁFICAS}

ALONSO-ALMEIDA, M.; RODRIGUEZ, M.; CORTEZ, K. y ABREU, J.L. (2012). "La responsabilidad social corporativa y el desempeño financiero: un análisis en empresas mexicanas que cotizan en bolsa". Contaduría y Administración, 57, pp. 53-77.

AMAT, O. (2000). Análisis de estados financieros: fundamentos y aplicaciones. Barcelona: Gestión 2000.

ANTUNES, R.B. y GALLARDO, D.A. (2016). "La relación entre la ISO 14001 y el desempeño financiero de las empresas cotizadas en el mercado bursátil en Portugal". $X V I I$ Encuentro AECA. Bragança, Portugal.

BEM, A.; PREDKIEWICZ, K.; PREDKIEWICZ, P. y UCIEKLAK-JEZ, P. (2014). "Determinants of Hospital's Financial Liquidity". Procedia Economics and Finance, 12, pp. 27-36.

BEM, A.; PREDKIEWICZ, K.; PREDKIEWICZ, P. y SIEDLECKI, R. (2015). "Profitability versus debt in hospital industry". European financial systems 2015. Proceedings of the 12th international scientific conference, pp. 20-7.

BENTO, W. (2013). "A Profitability Evaluation of America's Best Hospitals, 2000-2008". Decision Sciences, 44(6), pp. 1139-53.

CALVO, A. (2007). "Determinantes de la utilización de la información contable en lo hospital empresarial portugués". Conocimiento, innovación y emprendedores: camino al futuro. (pp. 989-1005).

CAMACHO, G.; PORTALES, L. y GARCÍA DE LA TORRE, C. (2013). "Herramientas de gestión de la RSE en América Latina". En RAUFFLET, E.; GARCÍA DE LA TORRE, C.; LOZANO, F.; BARRERA, E.: Responsabilidad Social Empresarial (pp. 1-13). México: Pearson.

CHARLO, M.J. y MOYA I. (2010). "El comportamiento financiero de las empresas socialmente responsables". Investigaciones Europeas de Dirección y Economía de la Empresa, 16(2), pp. 15-25.

CLEVERLEY, W. (1990). "Improving financial performance: a study of 50 hospitals". Hospital \& Health Services Administration, 35(2), pp. 173-87.

CORTÉS-FRANCH, I. y LÓPEZ-VALCÁRCEL, G. (2014). "Crisis económico-financiera y salud en España. Evidencia y perspectivas". Gaceta Sanitaria, 28(S1), pp. 1-6.

CORTÉS, A.M.; RAYO, S. y LARA, J. (2011). "Un Modelo Explicativo-Predictivo de la Rentabilidad Financiera de las Empresas en los Principales Sectores Económicos Españoles". XVI CONGRESO AECA. Granada.

CREIXANS-TENAS, J. y ARIMANY-SERRAT, N. (2016). "Gaudeixen de salut financera els hospitals privats catalans?" Intangible Capital, 12(1), pp. 198-222.

FRÍAS-ACEITUNO, J.V.; MARQUES, M.C. y RODRÍGUEZ-ARIZA, L. (2013). "Divulgación de información sostenible: ¿se adapta a las expectativas de la sociedad?" Revista Contabilidad: Spanish Accounting Rewiew, 16(2), pp. 147-158.

GINER, J.; LORENZO, R.M. y ABÁSOLO, I. (2005). "Análisis financiero de las empresas hospitalarias en Canarias: un estudio comparado en el conjunto español". Encuentros ALDE, pp. 1-19.

GÓMEZ, F. (2008). "Responsabilidad social corporativa y performance financiero: treinta y cinco años de investigación empírica en busca de un consenso". Principios: estudios de economía política, 11, pp. 5-24. 
INSTITUTO PARA EL DESARROLLO E INTEGRACIÓN DE LA SANIDAD. (2017). Sanidad Privada, aportando valor. Análisis de situación 2017. Madrid: IDIS.

JIMÉNEZ, J.; GARCÍA-GUTIÉRREZ, J.F. y BERMÚDEZ-TAMAYO, C. (2009). "Accesibilidad de sitios web sobre salud para mayores". Revista Española de Geriatría y Gerontología, 44(6), pp. 342-8.

JIMÉNEZ, M.A.; GESSA, A.; IRIMIA; A.I.; MORALES, S. y RUIZ, A. (2014). "Responsabilidad social corporativa (RSC) y rentabilidad financiera (RF) en el sector turístico" en Cultura, desarrollo y nuevas tecnologías". VII Jornadas de Investigación en Turismo, Sevilla, pp. 263-88.

LEE, M. y CHOI, M. (2011). "Difference of conflict levels of nurses and nurse-aids against doctors". Journal of the Korea Academia-Industrial, 12(11), pp. 4844-51.

LÓPEZ, G. (2009). Reflexiones sobre el futuro de la sanidad privada en España. Universitat Pompeu Fabra.

LUNA, L.; RESTREPO, J.E. y ZÚÑIGA, D. (2009). "Fluctuaciones macroeconómicas y comportamiento de las empresas en Chile". Economía Chilena, 12(2), pp. 83-95.

MARÍN-CALAHORRO, F. (2008). Responsabilidad Social Corporativa y comunicación. Madrid: Fragua.

MENEU, R; y ORTÚN, V. (2011). "Transparencia y buen gobierno en sanidad. También para salir de la crisis". Gaceta Sanitaria, 25(4), pp. 333-8.

MIRAS, M.M.; CARRASCO, A. y ESCOBAR, B. (2011). "Una revisión de los meta-análisis sobre responsabilidad social corporativa y rendimiento financiero". Revista de Estudios Empresariales. Segunda Época, 1, pp. 118-32.

NAVARRO, J.L. y GONZÁLEZ, J.M. (2006). "Responsabilidad social corporativa y crecimiento económico". Estudios de economía aplicada, 24(2), pp. 723-49.

NEVADO-GIL, M.T. y GALLARDO-VÁZQUEZ, D. (2016). "Información sobre Responsabilidad Social contenida en las páginas webs de los ayuntamientos. Estudio en la región del Alentejo". Revista española de documentación científica, 39(4), e150, pp. 1-17.

PINK, G.H.; DANIEL, I.; MCGILLIS, L. y MCKILLOP, I. (2007). "Selection of Key Financial Indicators: A Literature, Panel and Survey Approach". Law \& Governance, 11(3), pp. 87-96.

PRADO-LORENZO, J.M. y GARCÍA-SÁNCHEZ, I.M. (2010). "The role of the Board of Directors in disseminating relevant information on greenhouse gases". Journal of Business Ethics, 97(3), pp. 391-424.

RODRÍGUEZ-CALA, A.; CALLE-RODRÍGUEZ, C.; DURÁN-GARCIA, N. y ZÖLLER, B. (2015). "La responsabilidad social corporativa en los hospitales públicos de Cataluña: un estudio comparativo a través de las páginas web corporativas y la opinión de los responsables de RSC de los centros estudiados". Gest y Eval Cost Sanit, 16(2), pp. 193-205.

SATORRAS, M. (2002). La qualitat als centres sanitaris: experiènces a l'àrea de Barcelona. Barcelona: Edicions UPC.

SETÓ, D. y ANGLA, J. (2011). "La naturaleza de la relación entre la responsabilidad social de la empresa (RSE) y el resultado financiero". Revista Europea de Dirección y Economía de la Empresa, 20(4), pp.161-76.

VALENZUELA, L.; JARA-BERTIN, M. y VILLEGAS, F. (2015). "Prácticas de responsabilidad social, reputación corporativa y desempeño financiero". Revista de Administração de Empresas, 55(3), pp. 329-44. 